\title{
Relationship Between Diabetes Mellitus and Hemostasis: A Prothrombotic Condition
}

${ }^{1}$ Dilek Arpaci, ${ }^{2}$ Fatma Saglam, ${ }^{2}$ Didem Ozdemir, ${ }^{2}$ Reyhan Ersoy, ${ }^{2}$ Bekir Cakir

1. Sakarya University Education and Research Hospital, Department of Endocrinology, Sakarya, Turkey

2. Yildirim Beyazit University Ankara Ataturk Education and Research Hospital, Department of Endocrinology, Ankara, Turkey

\begin{abstract}
Background: Diabetes is very common disorder. Many studies have shown that patients with diabetes mellitus have incresing trombotic complications both arterial and venous trombosis. Bad control diabetes increases risk of trombosis. Recent reports have shown that shortened APTTs and increased fibrinogen indicate procoagulan situations. In this study we aimed to evaluate whether chronic hyperglisemia or bad control diabetes causes trombosis which is reflected by shortened APTTs and increased fibrinogen.
\end{abstract}

Materials and Methods: Our study included 349 patients with type 2 diabetes mellitus. They all underwent blood sampling APTT, PT, fibrinogen, fasting plasma glucose (FPG), postprandial plasma glucose (PPG), complete blood count $(\mathrm{CBC})$, serum lipids and HBA1C measurements. Among 349 patients whose APTT $<22 \mathrm{sec}$ and $\mathrm{PT}<10.5 \mathrm{sec}$ were determined. Patients were divided into two groups based on HBA1C levels as follows: regulated diabetic group (HBA1C $\leq 7.0 \%$ ) and disregulated diabetic group (HBA1C $>7.0 \%$ ). (Table 1)

Results: But there was no significant difference in terms of APTT $<22 \mathrm{sec}, \mathrm{PT}<10,5 \mathrm{sec}$ and fibrinogen levels between two groups.

Conclucion: APTT, PT and fibrinogen measurements are relatively inexpensive and are available. But they are not enough alone for evaluating hypercoaguable states in diabetes patients.

Key Words: Diabetes Mellitus, APTTs, fibrinogen. 
Table 1 Demographic and laboratory parameters evaluated in patients grouped according to their $\mathrm{HbA1C}$ levels.

\begin{tabular}{|c|c|c|c|}
\hline & \multicolumn{2}{|c|}{ HBA1C(\%) } & \multirow[t]{2}{*}{$\mathrm{P}$} \\
\hline & $\leq 7.0$ & $>7.0$ & \\
\hline $\mathrm{N}$ & 171 & 178 & \\
\hline Age(years) & $56(25-85)$ & $55.5(32-81)$ & 0.57 \\
\hline Gender & & & 0.193 \\
\hline Female & $55(32 \%)$ & $46(25 \%)$ & \\
\hline Male & $116(68 \%)$ & $132(75 \%)$ & \\
\hline BMI $(\mathrm{kg} / \mathrm{m} 2)$ & $25.8(19-28)$ & $25.76(18-28)$ & 0.50 \\
\hline APTT(s) & $26.11 \pm 6.5$ & $26.05 \pm 5.6$ & 0.60 \\
\hline APTT $<22 \mathrm{~s}$ & 16 & 16 & 0.47 \\
\hline PT(s) & $10.23 \pm 1.4$ & $10.24 \pm 1.3$ & 0.962 \\
\hline $\mathrm{PT}<10.5 \mathrm{~s}$ & 115 & 119 & 0.735 \\
\hline Fibrinogen(mg/dl) & $323.42(119-662)$ & $342.36(153-785)$ & 0.135 \\
\hline $\begin{array}{l}\text { Fibrinogen }>400 \\
\mathrm{mg} / \mathrm{dl}\end{array}$ & 26 & 40 & 0.529 \\
\hline Lipid profile & & & \\
\hline Trigliseride(mg/dl) & $133, \quad(43-437)$ & $174.6,(41-1568)$ & $\mathrm{P}=0.002$ \\
\hline LDL-C(mg/dl) & $105.6, \quad(31-251)$ & $107.6, \quad(27-206)$ & $\mathrm{P}=0.56$ \\
\hline HDL-C(mg/dl) & 47.7, (24-91) & $44.4,(21-146)$ & $\mathrm{P}<0.001$ \\
\hline $\begin{array}{l}\text { Postprandial plasma } \\
\text { glucose }(\mathrm{mg} / \mathrm{dl})\end{array}$ & $159, \quad(50-324)$ & $257.2, \quad(42-610)$ & $\mathrm{P}<0.001$ \\
\hline $\begin{array}{l}\text { Fasting plasma } \\
\text { glucose }(\mathrm{mg} / \mathrm{dl})\end{array}$ & $119,(77-386)$ & $128,(86-456)$ & $\mathrm{P}<0.001$ \\
\hline
\end{tabular}

